\title{
Locality of optimal lattice domain-wall fermions
}

\author{
Ting-Wai Chiu ${ }^{\text {a,b,c }}$ \\ a Institute for Advanced Study, Princeton, NJ 08540, USA \\ ${ }^{\mathrm{b}}$ Department of Physics, University of Washington, Seattle, WA 98195-1560, USA \\ ${ }^{\mathrm{c}}$ Department of Physics, National Taiwan University, Taipei, Taiwan 106, Taiwan
}

Received 16 November 2002; accepted 25 November 2002

Editor: M. Cvetič

\begin{abstract}
It is shown that the effective 4D lattice Dirac operator of optimal lattice domain-wall fermions with finite $N_{s}$ (in the fifth dimension) is exponentially local for sufficiently smooth gauge background.
\end{abstract}

(C) 2002 Elsevier Science B.V. All rights reserved.

PACS: 11.15.Ha; 11.30.Rd; 12.38.Gc

Keywords: Domain-wall fermions; Overlap Dirac operator; Zolotarev optimal rational polynomial

Recently, it has been shown that one can construct a lattice domain-wall fermion action such that the effective 4D lattice Dirac operator preserves the chiral symmetry optimally for any given finite $N_{s}$ in the fifth dimension [1].

Explicitly, the action of optimal lattice domain-wall fermions reads ${ }^{1}$

$$
\begin{aligned}
\mathcal{A}=\sum_{s, s^{\prime}=1}^{N_{s}} \sum_{x, x^{\prime}} \bar{\psi}(x, s) & \\
\times & {\left[\left(\omega_{s} D_{w}\left(x, x^{\prime}\right)+1\right) \delta_{s, s^{\prime}}\right.} \\
& \quad+\left(\omega_{s} D_{w}\left(x, x^{\prime}\right)-1\right) P_{-} \delta_{s^{\prime}, s+1} \\
& \left.\quad+\left(\omega_{s} D_{w}\left(x, x^{\prime}\right)-1\right) P_{+} \delta_{s^{\prime}, s-1}\right] \\
\times & \psi\left(x^{\prime}, s^{\prime}\right)
\end{aligned}
$$

\footnotetext{
E-mail address: twchiu@phys.ntu.edu.tw (T.-W. Chiu).

${ }^{1}$ In this Letter, we suppress the lattice spacings ( $a$ and $a_{5}$ ), as well as the Dirac and color indices, which can be restored easily.
}

$$
\begin{aligned}
-\frac{m_{q}}{2 m_{0}} \sum_{x, x^{\prime}}[ & \bar{\psi}(x, 1)\left(\omega_{1} D_{w}\left(x, x^{\prime}\right)-1\right) \\
& \times P_{+} \psi\left(x^{\prime}, N_{s}\right) \\
& +\bar{\psi}\left(x, N_{s}\right)\left(\omega_{N_{s}} D_{w}\left(x, x^{\prime}\right)-1\right) \\
& \left.\times P_{-} \psi\left(x^{\prime}, 1\right)\right]
\end{aligned}
$$

with boundary conditions

$$
\begin{aligned}
& P_{+} \psi(x, 0)=-\frac{m_{q}}{2 m_{0}} P_{+} \psi\left(x, N_{s}\right), \\
& P_{-} \psi\left(x, N_{s}+1\right)=-\frac{m_{q}}{2 m_{0}} P_{-} \psi(x, 1),
\end{aligned}
$$

and the quark fields with bare mass $m_{q}$ can be constructed from the left and right boundary modes

$$
\begin{aligned}
& q(x)=P_{-} \psi(x, 1)+P_{+} \psi\left(x, N_{s}\right), \\
& \bar{q}(x)=\bar{\psi}(x, 1) P_{+}+\bar{\psi}\left(x, N_{s}\right) P_{-} .
\end{aligned}
$$


Here $D_{w}$ is the $4 \mathrm{D}$ Wilson-Dirac operator with a negative parameter $\left(-m_{0}\right)$,

$$
\begin{gathered}
D_{w}=\sum_{\mu=1}^{4} \gamma_{\mu} t_{\mu}+W-m_{0}, \quad m_{0} \in(0,2), \\
t_{\mu}\left(x, x^{\prime}\right)=\frac{1}{2}\left[U_{\mu}(x) \delta_{x^{\prime}, x+\mu}-U_{\mu}^{\dagger}\left(x^{\prime}\right) \delta_{x^{\prime}, x-\mu}\right], \\
W\left(x, x^{\prime}\right)=\sum_{\mu=1}^{4} \frac{1}{2}\left[2 \delta_{x, x^{\prime}}-U_{\mu}(x) \delta_{x^{\prime}, x+\mu}\right. \\
\left.-U_{\mu}^{\dagger}\left(x^{\prime}\right) \delta_{x^{\prime}, x-\mu}\right],
\end{gathered}
$$

and the weights

$\omega_{s}=\frac{1}{\lambda_{\min }} \sqrt{1-\kappa^{\prime 2} \operatorname{sn}^{2}\left(v_{s} ; \kappa^{\prime}\right)}, \quad s=1, \ldots, N_{s}$,

where $\operatorname{sn}\left(v_{s} ; \kappa^{\prime}\right)$ is the Jacobian elliptic function with argument $v_{s}$ (3) and modulus $\kappa^{\prime}=\sqrt{1-\lambda_{\min }^{2} / \lambda_{\max }^{2}}$, $\left(\lambda_{\max }^{2}\right.$ and $\lambda_{\min }^{2}$ are the maximum and the minimum of the eigenvalues of $\left.H_{w}^{2}, H_{w}=\gamma_{5} D_{w}\right)$. The weights $\left\{\omega_{s}\right\}$ are obtained from the roots $\left(u_{s}=\omega_{s}^{-2}, s=\right.$ $\left.1, \ldots, N_{s}\right)$ of the equation

$\delta_{Z}(u)= \begin{cases}1-\sqrt{u} R_{Z}^{(n, n)}(u)=0, & N_{s}=2 n+1, \\ 1-\sqrt{u} R_{Z}^{(n-1, n)}(u)=0, & N_{s}=2 n .\end{cases}$

The argument $v_{s}$ in (2) is

$$
\begin{aligned}
v_{s}= & (-1)^{s-1} M \operatorname{sn}^{-1}\left(\sqrt{\frac{1+3 \lambda}{(1+\lambda)^{3}}} ; \sqrt{1-\lambda^{2}}\right) \\
& +\left[\frac{s}{2}\right] \frac{2 K^{\prime}}{N_{s}},
\end{aligned}
$$

where

$\lambda=\prod_{l=1}^{N_{s}} \frac{\Theta^{2}\left(\frac{2 l K^{\prime}}{N_{s}} ; \kappa^{\prime}\right)}{\Theta^{2}\left(\frac{(2 l-1) K^{\prime}}{N_{s}} ; \kappa^{\prime}\right)}$,

$M=\prod_{l=1}^{\left[\frac{N_{s}}{2}\right]} \frac{\operatorname{sn}^{2}\left(\frac{(2 l-1) K^{\prime}}{N_{s}} ; \kappa^{\prime}\right)}{\operatorname{sn}^{2}\left(\frac{2 l K^{\prime}}{N_{s}} ; \kappa^{\prime}\right)}$,

$K^{\prime}$ is the complete elliptic integral of the first kind with modulus $\kappa^{\prime}$, and $\Theta$ is the elliptic theta function. Note that $\lambda_{\max }^{-1} \leqslant \omega_{s} \leqslant \lambda_{\min }^{-1}$, since $\operatorname{sn}^{2}(;) \leqslant 1$.

After adding pseudofermions (Pauli-Villars fields) with fixed mass $m_{q}=2 m_{0}$ to the action (1), one can derive the effective 4D lattice Dirac operator for the internal quark loops

$$
D\left(m_{q}\right)=r\left(D_{c}+m_{q}\right)\left(1+r D_{c}\right)^{-1}, \quad r=\frac{1}{2 m_{0}},
$$

where

$r D_{c}=\frac{1+\gamma_{5} \mathcal{S}_{\mathrm{opt}}}{1-\gamma_{5} \mathcal{S}_{\mathrm{opt}}}$

$\mathcal{S}_{\mathrm{opt}}= \begin{cases}H_{w} R_{Z}^{(n, n)}\left(H_{w}^{2}\right), & N_{s}=2 n+1, \\ H_{w} R_{Z}^{(n-1, n)}\left(H_{w}^{2}\right), & N_{s}=2 n,\end{cases}$

and $R_{Z}\left(H_{w}^{2}\right)$ is the Zolotarev optimal rational approximation for the inverse square root of $H_{w}^{2}$,

$R_{Z}^{(n, n)}\left(H_{w}^{2}\right)=\frac{d_{0}}{\lambda_{\min }} \prod_{l=1}^{n} \frac{1+h_{w}^{2} / c_{2 l}}{1+h_{w}^{2} / c_{2 l-1}}$

and

$R_{Z}^{(n-1, n)}\left(H_{w}^{2}\right)=\frac{d_{0}^{\prime}}{\lambda_{\min }} \frac{\prod_{l=1}^{n-1}\left(1+h_{w}^{2} / c_{2 l}^{\prime}\right)}{\prod_{l=1}^{n}\left(1+h_{w}^{2} / c_{2 l-1}^{\prime}\right)}$

in which $h_{w}^{2}=H_{w}^{2} / \lambda_{\min }^{2}$, and the coefficients $d_{0}, d_{0}^{\prime}, c_{l}$ and $c_{l}^{\prime}$ are expressed in terms of elliptic functions [2] depending on $N_{s}$ and $\lambda_{\max }^{2} / \lambda_{\min }^{2}$.

In the limit $N_{s} \rightarrow \infty, \mathcal{S}_{\mathrm{opt}} \rightarrow H_{w}\left(H_{w}^{2}\right)^{-1 / 2}$, thus $r D_{c}$ (7) becomes chirally symmetric. Therefore, in the massless limit $\left(m_{q}=0\right)$ and $N_{s} \rightarrow \infty, D(6)$ is exactly equal to the overlap Dirac operator [3,4], and satisfies the Ginsparg-Wilson relation [5]

$D \gamma_{5}+\gamma_{5} D=2 D \gamma_{5} D$

This implies that the effective 4D Dirac operator of optimal DWF is exponentially local for sufficiently smooth gauge background, and is topologically-proper (i.e., with the correct index and axial anomaly), similar to the case of overlap Dirac operator. At finite $N_{s}$, the effective 4D lattice Dirac operator is exactly equal to the overlap Dirac operator with $\left(H_{w}\right)^{-1 / 2}$ approximated by Zolotarev optimal rational polynomial.

The valence quark propagator coupling to physical hadrons is

$\left(D_{c}+m_{q}\right)^{-1}=r\left(1-r m_{q}\right)^{-1}\left[D^{-1}\left(m_{q}\right)-1\right]$,

where $D^{-1}\left(m_{q}\right)$ can be computed via the 5-dimensional lattice Dirac operator of optimal DWF. 
In this Letter, we show that the effective 4D lattice Dirac operator (6) is exponentially local for sufficiently smooth gauge configurations. Since Eq. (6) can be rewritten as

$D\left(m_{q}\right)=r m_{q}+\left(1-r m_{q}\right) D$,

where

$D=r D_{c}\left(1+r D_{c}\right)^{-1}=\frac{1}{2}\left(1+\gamma_{5} \mathcal{S}_{\text {opt }}\right)$,

thus it suffices to assert the exponential locality of $D$ (11).

First, we rewrite (8) and (9) in partial fractions

$$
\begin{aligned}
& R_{Z}^{(n, n)}\left(H_{w}^{2}\right)=\left(1+h_{w}^{2} / c_{2 n}\right) \sum_{l=1}^{n} \frac{b_{l}}{1+h_{w}^{2} / c_{2 l-1}}, \\
& R_{Z}^{(n-1, n)}\left(H_{w}^{2}\right)=\sum_{l=1}^{n} \frac{b_{l}}{1+h_{w}^{2} / c_{2 l-1}},
\end{aligned}
$$

where

$$
\begin{aligned}
& c_{l}=\frac{\operatorname{sn}^{2}\left(\frac{l K^{\prime}}{N_{s}} ; \kappa^{\prime}\right)}{1-\operatorname{sn}^{2}\left(\frac{l K^{\prime}}{N_{s}} ; \kappa^{\prime}\right)}, \\
& b_{l}=\frac{1}{\lambda_{\min }} \frac{2 \lambda}{1+\lambda} \frac{1}{M} \frac{\prod_{i=1}^{n-1}\left(1-c_{2 l-1} / c_{2 i}\right)}{\prod_{i=1, i \neq l}^{n}\left(1-c_{2 l-1} / c_{2 i-1}\right)},
\end{aligned}
$$

$\lambda$ and $M$ are defined in (4) and (5), respectively.

Therefore, it suffices to examine the locality of each term in (12) and (13), by expanding each fraction in terms of orthogonal polynomials, similar to the approach used in [6]. Here I expand each fraction in term of Chebycheff polynomials of the first kind

$$
\begin{aligned}
& \frac{1}{1+h_{w}^{2} / c_{2 l-1}} \\
& =\frac{2 c_{2 l-1}}{(b-1) \sinh \theta_{l}}\left(1+2 \sum_{k=1}^{\infty} e^{-k \theta_{l}} T_{k}(z)\right),
\end{aligned}
$$

where

$$
\begin{aligned}
& b=\frac{\lambda_{\max }^{2}}{\lambda_{\min }^{2}} \\
& \theta_{l}=\cosh ^{-1}\left(\frac{b+1+2 c_{2 l-1}}{b-1}\right), \\
& z=\frac{b+1-2 h_{w}^{2}}{b-1} .
\end{aligned}
$$

The expansion (16) is valid provided that $\theta_{l} \neq 0$ (i.e., $\lambda_{\min }^{2}>0$ ), which is ensured by any gauge configuration satisfying [7]

$\forall$ plaquette $\|1-U(p)\|<\epsilon<\frac{1-\left|1-m_{0}\right|^{2}}{6(2+\sqrt{2})}$

since

$$
\begin{aligned}
& \left(\sqrt{1-6(2+\sqrt{2}) \epsilon}-\left|1-m_{0}\right|\right)^{2} \\
& \leqslant \lambda_{\text {min }}^{2}<\lambda_{\text {max }}^{2} \leqslant\left(\left|4-m_{0}\right|+4\right)^{2} .
\end{aligned}
$$

Because $h_{w}$ only has nearest neighbor coupling, it follows that $h_{w}^{2}(x, y)=0$ for all separations bigger than two lattice spacings, i.e., $\sum_{\mu}\left|x_{\mu}-y_{\mu}\right|>2 a$. Thus, for the Chebycheff polynomial $T_{k}(z)$ of degree $k,\left[T_{k}(z)\right](x, y)=0$ for $\sum_{\mu}\left|x_{\mu}-y_{\mu}\right|>2 k a$. Then, (16) gives

$$
\begin{aligned}
& \left(\frac{1}{1+h_{w}^{2} / c_{2 l-1}}\right)(x, y) \\
& \quad=\frac{2 c_{2 l-1}}{(b-1) \sinh \theta_{l}} \sum_{k=m}^{\infty} e^{-k \theta_{l}\left[T_{k}(z)\right](x, y),}
\end{aligned}
$$

where $m=\sum_{\mu}\left|x_{\mu}-y_{\mu}\right| / 2 a \geqslant 1$. Using the triangle inequality and the property $\left\|T_{k}(z)\right\| \leqslant 1$, we turn Eq. (22) into the inequality

$$
\begin{aligned}
& \left\|\left(\frac{1}{1+h_{w}^{2} / c_{2 l-1}}\right)(x, y)\right\| \\
& \leqslant \frac{2 c_{2 l-1}}{(b-1) \sinh \theta_{l}} \frac{e^{-m \theta_{l}}}{\left(1-e^{\left.-\theta_{l}\right)}\right.},
\end{aligned}
$$

and it follows that

$$
\begin{aligned}
\left\|\left(\sum_{l=1}^{n} \frac{b_{l}}{1+h_{w}^{2} / c_{2 l-1}}\right)(x, y)\right\| \\
\leqslant \frac{2}{b-1} \sum_{l=1}^{n} \frac{b_{l} c_{2 l-1}}{\sinh \theta_{l}} \frac{1}{\left(1-e^{\left.-\theta_{l}\right)}\right.} \\
\quad \times \exp \left(-\frac{\theta_{l}}{2 a} \sum_{\mu}\left|x_{\mu}-y_{\mu}\right|\right) \\
<\frac{2}{b-1}\left(\sum_{l=1}^{n} \frac{b_{l} c_{2 l-1}}{\sinh \theta_{l}} \frac{1}{\left(1-e^{-\theta_{l}}\right)}\right) \\
\quad \times \exp \left(-\frac{\theta_{1}}{2 a} \sum_{\mu}\left|x_{\mu}-y_{\mu}\right|\right),
\end{aligned}
$$


where the last inequality is due to the property $\left(\theta_{1}<\right.$ $\left.\theta_{2}<\cdots<\theta_{n}\right)$ from (18), since $\left(0<c_{1}<c_{3}<\cdots<\right.$ $\left.c_{2 n-1}\right)$ from (14). From (11)-(13), and (23), it follows that $D(11)$ is exponentially-local, and so is $D\left(m_{q}\right)$ (10).

Obviously, the above analysis also ensures that the overlap Dirac operator with $\left(H_{w}^{2}\right)^{-1 / 2}$ approximated by Zolotarev optimal rational polynomial is exponentially-local. Note that, in practice, the least upper bound of (23) is much smaller than that on the r.h.s. of (23). This implies that $D(x, y)$ is still exponentially local even for rough gauge configurations bounded by an $\epsilon$ much larger than its least upper bound in (20).

Further, it is straightforward to assert the differentiability of $D$ (11) with respect to the gauge field, as well as the exponential locality of $D$ in the presence of an isolated near-zero modes of $H_{w}^{2}$, thus the details can be omitted.

Finally, it is instructive to observe that the decay constant $\theta_{1}$ in (23) is a monotonic decreasing function of $N_{s}$. In the limit $N_{s} \rightarrow \infty, c_{1} \rightarrow 0$, and $\theta_{1}$ becomes the minimum,

$\theta=\cosh ^{-1}\left(\frac{b+1}{b-1}\right)=\cosh ^{-1}\left(\frac{\lambda_{\max }^{2}+\lambda_{\min }^{2}}{\lambda_{\max }^{2}-\lambda_{\min }^{2}}\right)$.

In other words, at any finite $N_{s}, D(x, y)$ is more localized than itself at $N_{s}=\infty$. In the limit $\omega_{s}=1$, the effective 4D lattice Dirac operator is equivalent to the overlap Dirac operator with polar approximation, and $c_{1}$ is replaced with $d_{1}=\tan ^{2}\left(\pi / 2 N_{s}\right)$, thus its decay constant $\left(\theta_{1}\right)$ is always smaller (i.e., less localized) than that of the optimal DWF, for any finite $N_{s}$.

For the conventional DWF with open boundary conditions, it has been estimated [8] that the effective 4D lattice Dirac operator at $N_{s}=\infty$ behaves as

$$
\begin{aligned}
& \left\|D_{\text {eff }}(x, y)\right\| \\
& \quad \lesssim\left(\sum_{\mu}\left|x_{\mu}-y_{\mu}\right|\right) \exp \left(-\frac{\tilde{\theta}}{2 a} \sum_{\mu}\left|x_{\mu}-y_{\mu}\right|\right) .
\end{aligned}
$$

However, for finite $N_{s}$, the decay constant of $D_{\text {eff }}(x, y)$ has not been obtained. Note that the factor $\left(\sum_{\mu}\left|x_{\mu}-y_{\mu}\right|\right)$ in $D_{\text {eff }}(x, y)$ makes it decay slower than purely exponential decay. Nevertheless, this is only an artifact due to the expansion [8] in terms of Chebycheff polynomials of the second kind. If one expands the fraction in terms of Chebycheff polynomials of the first kind as (16), then one obtains a purely exponential bound,

$$
\left\|D_{\text {eff }}(x, y)\right\| \lesssim \exp \left(-\frac{\tilde{\theta}}{2 a} \sum_{\mu}\left|x_{\mu}-y_{\mu}\right|\right) .
$$

\section{Acknowledgements}

This work was supported in part by National Science Council, ROC, under the grant numbers NSC912112-M002-025 and NSC-40004F, and it was completed during my stay at Princeton Institute for Advanced Study. I am grateful to The Institute for Advanced Study, in particular Steve Adler, for support and kind hospitality. I also thank Herbert Neuberger for discussions.

\section{References}

[1] T.W. Chiu, hep-lat/0209153.

[2] N.I. Akhiezer, Elements of the theory of elliptic functions, in: Translations of Mathematical Monographs, Vol. 79, American Mathematical Society, Providence, RI, 1990.

[3] H. Neuberger, Phys. Lett. B 417 (1998) 141.

[4] R. Narayanan, H. Neuberger, Nucl. Phys. B 443 (1995) 305.

[5] P.H. Ginsparg, K.G. Wilson, Phys. Rev. D 25 (1982) 2649.

[6] P. Hernandez, K. Jansen, M. Luscher, Nucl. Phys. B 552 (1999) 363.

[7] H. Neuberger, Phys. Rev. D 61 (2000) 085015.

[8] Y. Kikukawa, Nucl. Phys. B 584 (2000) 511. 